\title{
Single and three pulse photoselection of dye probes in nematic liquid crystals
}

\author{
D. A. Armoogum, J. Bryant ${ }^{\dagger}$, E. M. Monge and A. J. Bain* \\ Department of Physics and Astronomy, University College London, London WC1E 6BT, UK
}

\begin{abstract}
We present results of single and three pulse photoselection studies of the dynamics of a fluorescent probe (Oxazine 4) in the nematic phase of the liquid crystal 4-n-pentyl-4'-cyanobiphenyl (5CB). The combination of these techniques with time resolved fluorescence anisotropy measurements allows the unambiguous determination of the full angular motion of the probe together with the ground and excited state degrees of equilibrium (steady state) alignment. The restricted geometry imposed by the nematic host is seen to impart a marked anisotropy in $\theta$ and $\phi$ diffusion $\left(\gamma_{\varphi} / \gamma_{\theta} \sim 4\right)$.
\end{abstract}

Keywords: molecular probe, alignment, rotational diffusion, picosecond, fluorescence anisotropy, polarisation.

\section{INTRODUCTION}

In an ordered medium it is crucial to achieve a full resolution of the orientational (both $\theta$ and $\phi$ ) dynamics as the imposition of order gives rise to a marked departure from conventional (isotropic) molecular relaxation ${ }^{1-5}$. Molecular order has to date been probed using a range of optical processes including Raman scattering ${ }^{6}$, laser induced fluorescence ${ }^{7-9}$ and surface second harmonic generation ${ }^{10}$. Picosecond fluorescence anisotropy studies of molecular probes have been shown to yield useful information on molecular order, orientational dynamics and photophysical processes in both molecular and biomolecular systems ${ }^{1,11}$. Recent work in our group has shown that it is possible to obtain information on the order and full angular motion of a fluorescent probe in an ordered environment using single pulse variable photoselection and picosecond polarised fluorescence ${ }^{2-4}$. Here the variation of the excitation (linear) polarisation gives rise to the creation of varying degrees of (non-equilibrium) cylindrically symmetric and asymmetric probe alignment. Analysis of the subsequent fluorescence anisotropy signals yields the ground and excited state degrees of molecular alignment together with the intrinsic rates for $\theta$ and $\phi$ diffusion in the laboratory axis system. This approach has been highly successful in the study of systems exhibiting moderate probe alignment where the departure from (otherwise) isotropic diffusion dynamics is well described by first order perturbation theory ${ }^{3}$. In single pulse photoselection the creation of the maximum probe alignment orthogonal to the laboratory $\mathrm{Z}$ axis (in the case of liquid crystals the nematic director) is achieved with an excitation polarisation angle of $90^{\circ 4}$. This invariably leads to the creation of both cylindrically symmetric and asymmetric degrees of excited state alignment and whose joint evolution is reflected in the measured fluorescence anisotropy. The use of three beam photoselection which involves the net photoselection resulting from three orthogonally polarised ( $\mathrm{Z}$ plus $\mathrm{X}$ and $\mathrm{Y}$ ), temporally coincident laser pulses allows for the creation of cylindrically symmetric but orthogonal probe alignment ${ }^{12}$. A combination of these techniques affords the unequivocal experimental determination of probe alignment dynamics within a nematic host.

Defining the measurement direction perpendicular to the $\mathrm{Z}$ axis as $\mathrm{X}$, the probe fluorescence anisotropy $\mathrm{R}(\mathrm{t})$ at time $\mathrm{t}$ following the application of an ultrashort pulse sequence (or single pulse) is given by ${ }^{1}$

$$
R(t)=\frac{\frac{1}{\sqrt{5}}\left\langle\alpha_{20}^{e x}(t)\right\rangle-\frac{1}{\sqrt{30}}\left\{\left\langle\alpha_{22}^{e x}(t)\right\rangle+\left\langle\alpha_{2-2}^{e x}(t)\right\rangle\right\}}{1+\frac{2}{\sqrt{30}}\left\{\left\langle\alpha_{22}^{e x}(t)\right\rangle+\left\langle\alpha_{2-2}^{e x}(t)\right\rangle\right\}}
$$

\footnotetext{
* Corresponding author, email a.bain@ucl.ac.uk

${ }^{\dagger}$ Current address: Solexa Ltd, Chesterfield Research Park, Little Chesterfield, nr Saffron Walden, Essex CB10 1XL, UK.
} 
where $\left\langle\alpha_{20}^{e x}(t)\right\rangle$ and $\left\{\left\langle\alpha_{22}^{e x}(t)\right\rangle+\left\langle\alpha_{2-2}^{e x}(t)\right\rangle\right\}$ are the time dependent cylindrically symmetric and asymmetric degrees of excited state alignment. Their initial values are dependent on the photoselection process and the degree of order in the ground state. From symmetry considerations to first order the diffusion dynamics in an aligned medium should be linear ${ }^{3}$. For a medium exhibiting global cylindrical symmetry, these are expected to follow

$$
\begin{aligned}
& \left\langle\alpha_{20}^{e x}(t)\right\rangle=\left\{\left\langle\alpha_{20}^{e x}(0)\right\rangle-\left\langle\alpha_{20}^{e x}(s s)\right\rangle\right\} \exp \left(-\frac{t}{\tau_{20}}\right)+\left\langle\alpha_{20}^{e x}(s s)\right\rangle \\
& \left\{\left\langle\alpha_{22}^{e x}(t)\right\rangle+\left\langle\alpha_{2-2}^{e x}(t)\right\rangle\right\}=\left\{\left\langle\alpha_{22}^{e x}(0)\right\rangle+\left\langle\alpha_{2-2}^{e x}(0)\right\rangle\right\} \exp \left(-\frac{t}{\tau_{22}}\right)
\end{aligned}
$$

where (ss) denotes the equilibrium or steady state value of the probe alignment. The cylindrically symmetric and asymmetric alignment rates $\tau_{20}$ and $\tau_{22}$ are related to the $\theta$ and $\phi$ diffusion rates in the laboratory frame $D_{\perp}^{L A B}$ and $D_{\|}^{L A B}$ respectively through

$$
\begin{aligned}
& 1 / \tau_{20}=6 D_{\perp}^{L A B} \\
& 1 / \tau_{22}=2 D_{\perp}^{L A B}+4 D_{\|}^{L A B}
\end{aligned}
$$
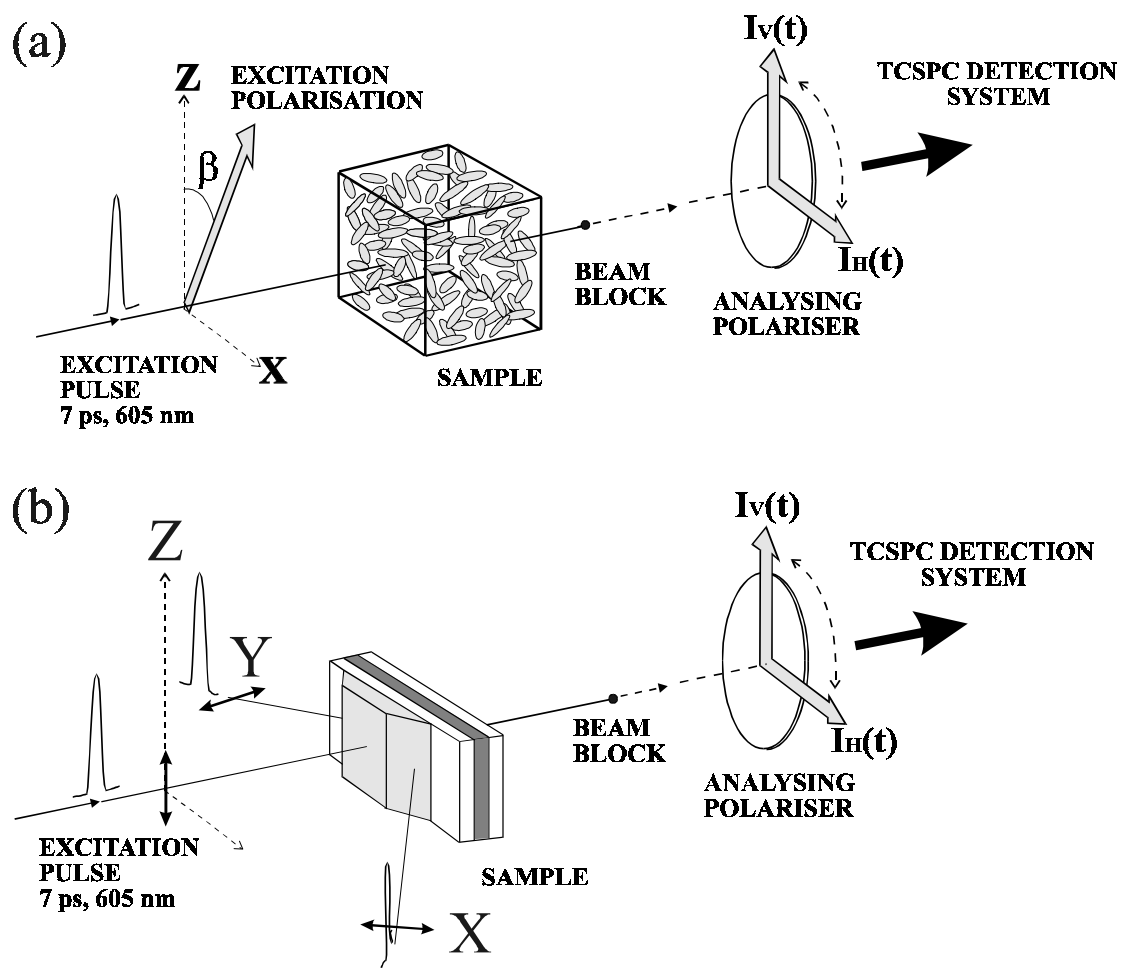

Figure 1. (a) A schematic representation of the experimental arrangement and $180^{\circ}$ excitation-detection geometry for single pulse polarised photoselection. The excitation polarisation angle $\beta$ is varied to create varying degrees of cylindrically symmetric and asymmetric excited state alignment. (b) Experimental arrangement used for three beam photoselection. The excitation from three orthogonally polarised coincident pulses results in a cylindrically symmetric excited state; variation of the $\mathrm{Z}$ polarised beam intensity relative to the (equal) $\mathrm{X}$ and $\mathrm{Y}$ beam intensities allows the cylindrically symmetric alignment to be controlled. Prism coupling is required to ensure the necessary $\pm 45^{\circ}$ propagation angles for the horizontally polarised pulses. 


\section{SINGLE PULSE PHOTOSELECTION}

The experimental arrangement for single pulse photoselection using a variable linear polarisation is shown in Fig 1a. The excitation polarisation angle $\beta$ is tuneable between $0^{\circ}$ to $90^{\circ}$ resulting in a transition probability of the form,

$$
W_{a b s}^{l a b}(\theta, \phi, t)=\left|\mu_{a}\right|^{2} \frac{(4 \pi)^{\frac{1}{2}}}{3}\left[Y_{00}(\theta, \phi)+\frac{2}{\sqrt{5}} \sum_{Q} d_{Q 0}^{2}(-\beta) Y_{2 Q}(\theta, \phi)\right]
$$

In a medium with an axis of cylindrical symmetry parallel to the laboratory Z-axis the degrees of initial excited state alignment are given $b^{3}$

$$
\left\langle\alpha_{2 Q}^{e x}(0)\right\rangle=\frac{\sum_{K^{\prime} Q^{\prime}}\left\langle\alpha_{K^{\prime} Q^{\prime}}^{g s}(s s)\right\rangle\left\langle 2 Q\left[Y_{00}(\theta, \phi)+\frac{2}{\sqrt{5}} \sum_{Q^{\prime}} d_{0 Q}^{2}(-\beta) Y_{2 Q^{\prime \prime}}(\theta, \phi)\right] \| K^{\prime} 0\right\rangle}{\sum_{K^{\prime} Q^{\prime}}\left\langle\alpha_{K^{\prime} Q^{\prime}}^{g s}(s s)\right\rangle\left\langle 00\left[Y_{00}(\theta, \phi)+\frac{2}{\sqrt{5}} \sum_{Q^{\prime}} d_{0 Q}^{2}(-\beta) Y_{2 Q^{\prime \prime}}(\theta, \phi)\right] \mid K^{\prime} 0\right\rangle}
$$

where $\left\langle\alpha_{K^{\prime} Q^{\prime}}^{g s}(s s)\right\rangle$ are the normalised steady state moments of the ground state distribution function $\left(\left\langle\alpha_{00}^{g s}(s s)\right\rangle=1\right)$. Evaluating matrix elements yields

$$
\begin{aligned}
& \frac{\left\langle\alpha_{20}^{e x}(0)\right\rangle}{\sqrt{5}}=\frac{\left[\frac{3 \cos ^{2} \beta-1}{5}+\frac{\left\langle\alpha_{20}^{g s}(s s)\right\rangle}{\sqrt{5}}\left[1+\frac{2}{7}\left(3 \cos ^{2} \beta-1\right)\right]+\frac{6\left\langle\alpha_{40}^{g s}(s s)\right\rangle}{35}\left(3 \cos ^{2} \beta-1\right)\right]}{\left[1+\frac{\left(3 \cos ^{2} \beta-1\right)}{\sqrt{5}}\left\langle\alpha_{20}^{g s}(s s)\right\rangle\right]} \\
& \frac{\left\langle\alpha_{22}^{e x}(0)\right\rangle+\left\langle\alpha_{2-2}^{e x}(0)\right\rangle}{\sqrt{30}}=\frac{\sin ^{2} \beta}{5}\left[1-\frac{10\left\langle\alpha_{20}^{g s}(s s)\right\rangle}{7 \sqrt{5}}+\frac{\left\langle\alpha_{40}^{g s}(s s)\right\rangle}{7}\right] /\left[1+\frac{\left(3 \cos ^{2} \beta-1\right)}{\sqrt{5}}\left\langle\alpha_{20}^{g s}(s s)\right\rangle\right]
\end{aligned}
$$

Variation of the excitation polarisation angle $\beta$ from 0 to $90^{\circ}$ allows the preparation of a range of initial degrees of excited state alignment with increasing cylindrical asymmetry as $\beta$ approaches $90^{\circ}$. This is accompanied by a decrease in $\left\langle\alpha_{20}^{e x}(0)\right\rangle$ which will tend, depending on the ground state order, towards a negative value as $\beta$ nears $90^{\circ}$. The variation of the cylindrically symmetric and asymmetric contributions to the excited state alignment of oxazine 4 in nematic 5CB with excitation polarisation angle $\beta$ is shown in figure 2 , with those for an isotropic system shown for comparison.

Observation of the alignment dynamics for an excitation polarisation angle of $\beta=0^{\circ}$ will clearly reveal the $\theta$ diffusion dynamics of probe molecules aligned predominantly along the laboratory Z-axis. For single beam photoselection (in a positively aligned medium) the observation of $\theta$ diffusion for probe molecules aligned orthogonally to $\mathrm{Z}$ is invariably convoluted with the asymmetric alignment dynamics. In a highly ordered environment such as an aligned mesophase of a liquid crystal it is possible that the diffusion dynamics may well not be linear and the simple picture of alignment relaxation above described in equation (2) will not hold. In these circumstances as clear an experimental picture of the full alignment dynamics is highly desirable. To this end the ability to control the initial photoselected excited state alignment whilst retaining an axis of cylindrical symmetry is highly desirable. This novel approach to alignment control in polarised fluorescence experiments has recently been demonstrated and uses the net photoselection produced by $\mathrm{Z}, \mathrm{X}$ and $\mathrm{Y}$ polarised excitation pulses ${ }^{11}$. 


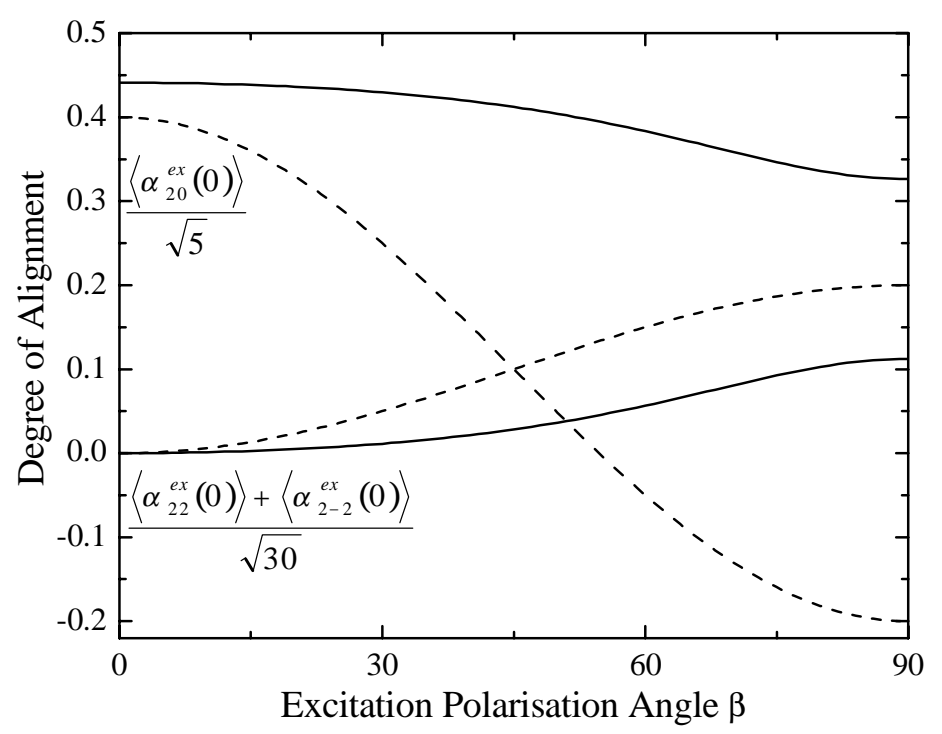

Figure 2. Excitation polarisation dependence of the cylindrically symmetric and asymmetric contributions to the initial fluorescence anisotropy for oxazine 4 in 5CB (solid lines) and for an isotropic system (dashed lines). As the excitation polarisation angle $\beta$ is increased from $0^{\circ}$, increasing cylindrical asymmetry is introduced into the excited state alignment.

\section{THREE PULSE POLARISED PHOTOSELECTION}

The background theory for three beam photoselection has been described in detail elsewhere ${ }^{11}$. The experimental arrangement for three pulse polarised photoselection is shown in fig $1 \mathrm{~b}$. We have shown in previous work that the implementation of coincident equal intensity $\mathrm{X}$ and $\mathrm{Y}$ polarised beams with propagation angles of $\pm 45^{\circ}$ to the collinear $\mathrm{Z}$ polarised beam photoselects an excited state with no cylindrically asymmetric alignment ${ }^{11}$. For single photon transitions within the mutual excitation volume the net excitation operator is given by an intensity weighted sum of the individual transition probabilities

$W_{a b s}^{l a b}(\theta, \phi, t) \propto\left|\vec{\mu}_{a}\right|^{2} \frac{\sqrt{4 \pi}}{3}\left[Y_{00}\left(\theta_{I}, \phi_{I}\right)+\frac{2}{\sqrt{5}} B(I) Y_{20}\left(\theta_{I}, \phi_{I}\right)\right]$

where $\mathrm{B}(\mathrm{I})$ is given by the ratio of the input intensities,

$B(I)=\frac{I_{Z}(t)-\frac{1}{2}\left[I_{X}(t)+I_{Y}(t)\right]}{I_{Z}(t)+I_{X}(t)+I_{Y}(t)}$

where $I_{X}$ and $I_{Y}$ represent the equal intensity horizontally polarised excitation beams that propagate at $\pm 45^{\circ}$ to the $Z$ polarised beam. By varying the ratio of $I_{X}$ and $I_{Y}$ to $I_{Z}, B(I)$ can be varied from $1\left(I_{X}=I_{Y}=0\right)$ to -0.5 ( $\left.I_{Z}=0\right)$. For an isotropic medium this leads to an initial excited state anisotropy range tuneable between 0.4 to -0.5 . Typical initial excited state orientational distribution probabilities $P^{e x}(\theta, \phi, 0)$ arising from three beam photoselection for an isotropic system as beam ratio $\mathrm{B}(\mathrm{I})$ is varied from 1 to -0.5 are shown in figure 3 . 


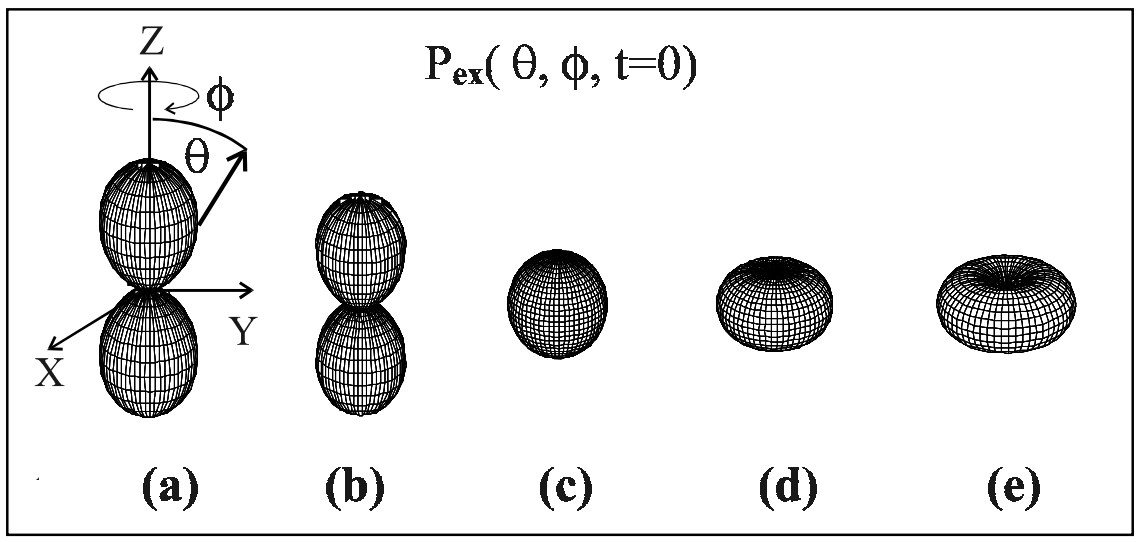

Figure 3. In an isotropic medium, three beam photoselection allows for the control of excited state alignment whilst retaining cylindrical symmetry as beam ratio $B(I)$ is varied. For $B(I)=1$, an excited state orientational distribution (a) is formed which yields an initial fluorescence anisotropy of 0.4 . For $\mathrm{B}(\mathrm{I})=-0.5$, an excited state orientational distribution (e) is formed which yields an initial fluorescence anisotropy of -0.5 . Although cylindrical symmetry is retained in the experiments with oxazine 4 in nematic $5 \mathrm{CB}$, isotropic and negatively aligned distributions such as in (c)-(e) are not observed, due to the intrinsic positive steady state alignment.

\subsection{Application to an aligned medium}

For an anisotropic medium the degree of excited state alignment is not only dependent upon the photoselection process but also upon the intrinsic alignment of the ordered ground state. In the presence of an axially symmetric ground state the initial fluorescence anisotropy is given by

$R(0)=\frac{\left\langle\alpha_{20}^{e x}(0)\right\rangle}{\sqrt{5}}=\frac{\frac{k-1}{A}+(2 k+1)\left[\frac{\left\langle\alpha_{20}^{g s}(s s)\right\rangle}{\sqrt{5}}+B(I)\left(\frac{2}{5}+\frac{4}{7} \frac{\left\langle\alpha_{20}^{g s}(s s)\right\rangle}{\sqrt{5}}+\frac{12}{35}\left\langle\alpha_{40}^{g s}(s s)\right\rangle\right)\right]}{\frac{k+2}{A}+2(k-1) B(I) \frac{\left\langle\alpha_{20}^{g s}(s s)\right\rangle}{\sqrt{5}}}$

where $\mathrm{k}$ and $\mathrm{A}$ are experimental correction factors (see section 4.1.1)

\section{EXPERIMENTAL REALISATION}

\subsection{Procedure}

A schematic representation of the experimental apparatus is shown in fig $1 \mathrm{~b}$. The oxazine 4 doped (c.a. $\left.10^{-4} \mathrm{M}\right)$ nematic $5 \mathrm{CB}$ was held in $100 \mu \mathrm{m}$ thick quartz cells. To provide a homogeneous alignment for the liquid crystal environment, the cells were coated with a surfactant (polyvinyl alcohol solution) and rubbed mechanically. A cell path length of 10 qum was chosen because it falls within the $300 \mu \mathrm{m}$ range over which homogeneous alignment persists ${ }^{13}$. The sample was contacted by index matching fluid to a coupling prism, fluorescence collection and analysis utilised a collinear geometry with respect to the normally incident Z-polarised excitation pulse $(605 \mathrm{~nm}, 7 \mathrm{ps})$. Fluorescence was detected and recorded using time correlated single photon counting (TCSPC) electronics. Particulars of sample preparation, the TCSPC system and associated components have been described in detail elsewhere ${ }^{4,14}$. 
Two preliminary measurements were made using a single pulse at $0^{\circ}$ incidence with excitation angle $\beta=0^{\circ}$ and $\beta=90^{\circ}$. The initial values of the two fluorescence anisotropies can be used to determine the $K=2$ and $K=4$ moments of the ground state distribution. In order to determine accurately these and other fluorescence observables it is necessary to take account of the alteration of the fluorescence anisotropy by the ordered sample and optical system, this results in the determination of two parameters and is described below.

\subsubsection{Corrections to the fluorescence anisotropy}

In a birefringent medium the vertical and horizontally polarised components of the fluorescence anisotropy are unequally affected by local field effects and differential reflection losses at the boundary between the sample the cell wall $^{15}$. Application of these considerations to emission from a molecular probe in a nematic host we obtain ${ }^{16}$ the anisotropy correction factor $\mathrm{k}$ given by,

$$
k(\lambda, T)=\left(\frac{n_{e}}{n_{o}}\right)\left(\frac{n_{o}+n_{g}}{n_{e}+n_{g}}\right)^{2}\left(\frac{n_{e}^{2}+2}{n_{o}^{2}+2}\right)
$$

In this analysis $\mathrm{k}$ is uniquely determined by the extraordinary and ordinary indices of refraction of the medium $\left(n_{e}\right.$ and $n_{o}$ ) and the refractive index of the sample cell wall $n_{g}$. The temperature and wavelength dependence of these for 5CB are well known ${ }^{17}$, however correction for the change in the nematic isotropic phase transition and averaging over the fluorescence emission spectrum is necessary ${ }^{14}$. We have recently shown that it is possible to determine $\mathrm{k}$ independently by a series of fluorescence lifetime and anisotropy measurements ${ }^{16,18}$, the values yielded by both methods are in general agreement. For oxazine 4 in $5 \mathrm{CB}$ at $\mathrm{T}=25^{\circ} \mathrm{C} \mathrm{k}=1.12463$.

The second correction factor labelled the A parameter takes into account the reduction in the fluorescence anisotropy from theoretical values due the possible contribution of a number of molecular and experimental factors. Principally these are the non-parallelism of absorption and emission transition dipole moments ${ }^{3}$, depolarisation due arising from sample concentration and path length effects ${ }^{19}$ and depolarisation by the collection optics. The A parameter is determined by the departure of the initial fluorescence anisotropy from its theoretical value in an isotropic sample ${ }^{4}$. For liquid crystalline media the sample is raised to the a few degrees above the nematic isotropic phase transition temperature where departure of $\mathrm{R}(0, \beta)$ from theory is then determined ${ }^{14}$. For oxazine 4 in $5 \mathrm{CB}$ this approach yielded a value for A of 0.881 .

\subsubsection{Prism Coupling}

In order to achieve the conditions whereby net cylindrical symmetry is maintained by three pulse photoselection, it is necessary for the horizontally polarised beams to propagate through the sample at $\pm 45^{\circ}$ to the $\mathrm{Z}$ polarised excitation pulse. From Snell's Law for propagation at $45^{\circ}$ to the normal the incident light angle $\xi_{\text {air }}$ at the air glass interface for a sample must satisfy

$$
\sin \xi_{\text {air }}=n_{\text {sample }} / \sqrt{2}
$$

It is clear therefore that $n_{\text {sample }} \leq \sqrt{ } 2$, however in highly ordered media such as $5 C B n_{\text {sample }}$ is greater than $\sqrt{ } 2$, so prism coupling is necessary to achieve $45^{\circ}$ sample propagation. Details of this are described elsewhere ${ }^{12}$. 


\subsubsection{Propagation angle determination.}

The correct propagation angle of $45^{\circ}$ for the $\mathrm{X}$ and $\mathrm{Y}$ polarised beams can be established from measurements of the initial anisotropy relation for horizontally polarised excitation $\left(\beta=90^{\circ}\right)$ with varying external propagation angle $\xi$. The variation of $\mathrm{R}_{\mathrm{H}}$ with $\xi$ is given by ${ }^{12}$,

$$
R_{H}(0, \xi)=\frac{\frac{k-1}{A}\left[1-\frac{\left\langle\alpha_{20}^{g s}\right\rangle}{\sqrt{5}}\right]+(2 k+1)\left[-\frac{1}{5}+\frac{5}{7} \frac{\left\langle\alpha_{20}^{g s}\right\rangle}{\sqrt{5}}-\frac{6}{35}\left\langle\alpha_{40}^{g s}\right\rangle\right]-\frac{3}{5} \cos (2 \xi)\left[1-\frac{10}{7 \sqrt{5}}\left\langle\alpha_{20}^{g s}\right\rangle-\frac{\left\langle\alpha_{40}^{g s}\right\rangle}{7}\right]}{\frac{k+2}{A}\left[1-\frac{\left\langle\alpha_{20}^{g s}\right\rangle}{\sqrt{5}}\right]+2(k-1)\left[-\frac{1}{5}+\frac{5}{7} \frac{\left\langle\alpha_{20}^{g s}\right\rangle}{\sqrt{5}}-\frac{6}{35}\left\langle\alpha_{40}^{g s}\right\rangle\right]+\frac{6}{5} \cos (2 \xi)\left[1-\frac{10}{7 \sqrt{5}}\left\langle\alpha_{20}^{g s}\right\rangle-\frac{\left\langle\alpha_{40}^{g s}\right\rangle}{7}\right]}
$$

The predicted variation in $\mathrm{R}_{\mathrm{H}}$ with propagation angle $\xi$ for oxazine 4 in $5 \mathrm{CB}$ is shown in figure $4,45^{\circ}$ propagation in the sample corresponding to an initial anisotropy of 0.325 . The angle of incidence of the horizontally polarised beam was varied until the target anisotropy of 0.325 was reached. The second horizontally polarised beam was similarly aligned and care was taken to ensure the three beams were spatially and temporally overlapped in the sample. Fluorescence anisotropy measurements were then made with varying values of beam intensity ratio B(I) from 1 [Z-polarised excitation] to -0.5 [ $\mathrm{X}$ and $\mathrm{Y}$ polarised excitation].

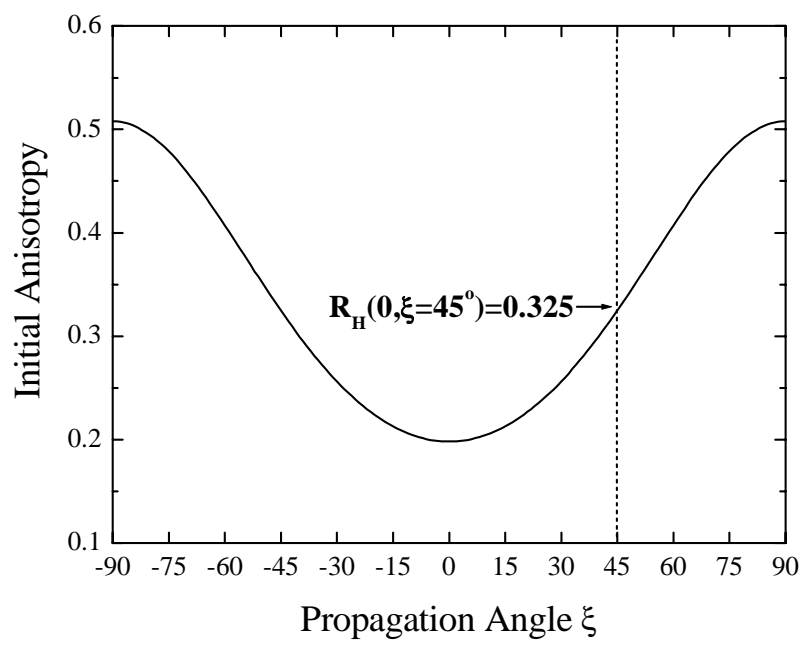

Figure 4. A plot of the variation in the initial anisotropy with angle of incidence $R_{H}(0, \xi)$ for oxazine 4 in $5 \mathrm{CB}$ predicted by the ground state moments. The predicted (target) initial anisotropy value for $45^{\circ}$ propagation is 0.325 . 


\section{RESULTS}

Fluorescence anisotropy decays following three beam photoselection for oxazine 4 in $5 \mathrm{CB}$ are shown in figure 5 . All the decays can be fitted to a single exponential lifetime, which is in excellent agreement (within experimental uncertainties) with the lifetime obtained for a single beam photoselection experiment using vertically polarised light. The single beam anisotropy decay with an excitation polarisation angle $\beta=90^{\circ}$ for oxazine 4 is included for comparison.

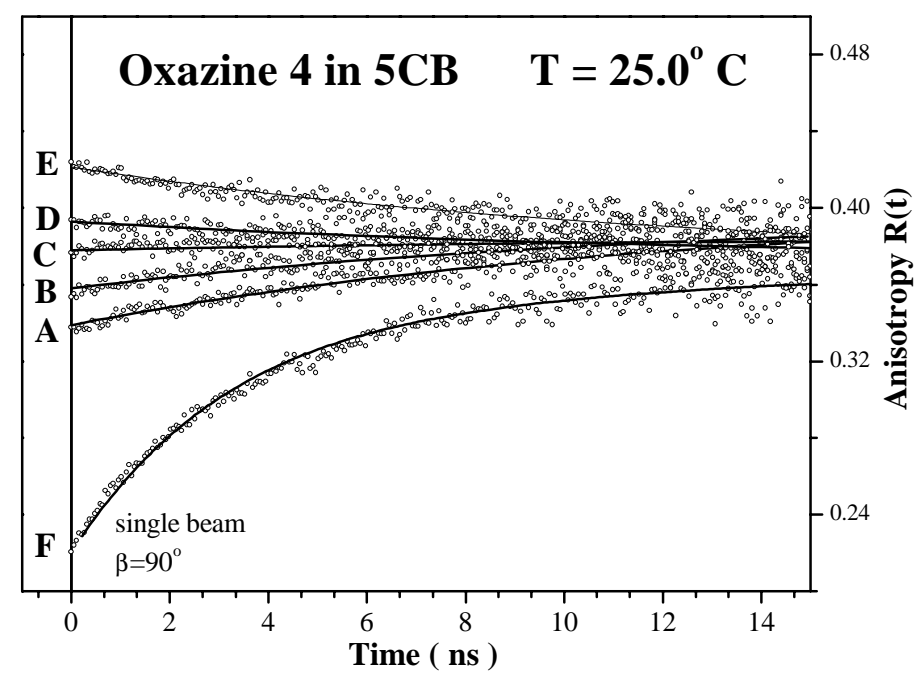

Figure 5. Three beam anisotropy decays (curves A to E) obtained for Oxazine 4 in $5 \mathrm{CB}\left(5 \times 10^{-4} \mathrm{M}\right)$, with a single beam $\beta=90^{\circ}$ anisotropy decay (curve F) shown for comparison. The intensity ratio $\mathrm{B}(\mathrm{I})$ is varied from -0.5 ( $\mathrm{X}$ and $\mathrm{Y}$ polarised photoselection) through to 1 ( $\mathrm{Z}$ polarised photoselection).

\begin{tabular}{|c|c|c|}
\hline Oxazine 4 curve label & Beam ratio B(I) & $\tau_{20}(\mathrm{~ns})$ \\
\hline $\mathrm{A}$ & -0.500 & $11.9 \pm 0.3$ \\
\hline $\mathrm{B}$ & 0.096 & $12.0 \pm 0.5$ \\
\hline $\mathrm{C}$ & 0.477 & $12.5 \pm 0.2$ \\
\hline $\mathrm{D}$ & 0.738 & $12.2 \pm 0.9$ \\
\hline $\mathrm{E}$ & \multicolumn{2}{|c|}{ Single pulse photoselection, $\beta=0^{\circ}: \mathrm{B}(\mathrm{I})=1, \tau_{20}=12.06 \pm 1.15 \mathrm{~ns}$} \\
\hline $\mathrm{F}$ & Single pulse photoselection, $\beta=90^{\circ}: \tau_{22}=4.04 \pm 0.01 \mathrm{~ns}$ \\
\hline
\end{tabular}

Table 1 . The anisotropy decays for oxazine 4 in 5CB yield (within experimental uncertainty) the same corrected single exponential lifetime as that obtained for single beam Z-polarised excitation. 
The three beam photoselection experiment provides a variable degree of alignment selectivity whilst retaining cylindrical symmetry. The tuning range of initial anisotropy depends upon the value of beam ratio $\mathrm{B}$ (I) for a given set of ground state parameters and correction factors. The predicted maximum and minimum degrees of initial alignment can be determined from equation (10) where $\mathrm{B}(\mathrm{I})$ is 1 and -0.5 respectively: this gives,

$$
\begin{aligned}
& R(0, \max )=\left[\frac{k-1}{A}+(2 k+1)\left[\left\langle P_{2}\right\rangle+\left(\frac{2}{5}+\frac{4}{7}\left\langle P_{2}\right\rangle+\frac{36}{35}\left\langle P_{4}\right\rangle\right)\right]\right] /\left[\frac{k+2}{A}+2(k-1)\left\langle P_{2}\right\rangle\right] \\
& R(0, \min )=\left[\frac{k-1}{A}+(2 k+1)\left[\left\langle P_{2}\right\rangle-\left(\frac{1}{5}+\frac{2}{7}\left\langle P_{2}\right\rangle+\frac{18}{35}\left\langle P_{4}\right\rangle\right)\right]\right] /\left[\frac{k+2}{A}-(k-1)\left\langle P_{2}\right\rangle\right]
\end{aligned}
$$

where $\left\langle\mathrm{P}_{2}\right\rangle$ and $\left\langle\mathrm{P}_{4}\right\rangle$ are the more commonly used $2^{\text {nd }}$ and $4^{\text {th }}$ rank order parameters used to describe cylindrically symmetric samples ${ }^{20}$ these are related to the alignment moments through

$$
\left\langle P_{K}\right\rangle=\left\langle\alpha_{K 0}\right\rangle / \sqrt{2 K+1}
$$

\begin{tabular}{|c|c|c|c|}
\hline OXAZINE 4, $\mathrm{T}=25^{\circ} \mathrm{C}$ & $\mathrm{B}(\mathrm{I})$ & $\mathrm{R}_{\text {predicted }}$ & $\mathrm{R}_{\text {observed }}$ \\
\hline $\begin{array}{l}\langle\mathrm{P} 2\rangle=0.3949 \\
\langle\mathrm{P} 4\rangle=-0.2245\end{array}$ & 1 & 0.42 & $0.42 \pm 0.01$ \\
\hline $\begin{aligned} \mathrm{k} & =1.12463 \\
\mathrm{~A} & =0.861\end{aligned}$ & -0.5 & 0.33 & $0.32 \pm 0.01$ \\
\hline
\end{tabular}

The tuning range of initial anisotropy measured for oxazine 4 in excellent accordance (i.e. within experimental error) with the predicted maximum and minimum initial anisotropy values, as shown in table 2 .

Table 2. The ground state parameters obtained from the single beam $\beta=0^{\circ}$ and $90^{\circ}$ curves predict the initial anisotropy tuning range that should be possible using three beam photoselection. The observed tuning range is in excellent agreement with predictions for oxazine 4 in $5 \mathrm{CB}$.

The symmetric and asymmetric alignment relaxation times $\left(\tau_{20}\right.$ and $\left.\tau_{22}\right)$ can be determined by fitting the single photon photoselection anisotropy decays for $\beta=0^{\circ}$ and $\beta=90^{\circ}$. Including correction factors, the fitting function for the anisotropy decays took the following form:

$$
R(\beta, t)=\left[\frac{k-1}{A}+\frac{\alpha_{20}(2 k+1)}{\sqrt{5}}-3 \alpha_{A S}\right] /\left[\frac{k+2}{A}+\frac{\alpha_{20}(2 k-2)}{\sqrt{5}}+6 \alpha_{A S}\right]
$$

where

$$
\frac{\alpha_{20}}{\sqrt{5}}=\left[\frac{\frac{3 \cos ^{2} \beta-1}{5}+\left\langle P_{2}\right\rangle\left(1+\frac{2\left(3 \cos ^{2} \beta-1\right)}{7}\right)+\left\langle P_{4}\right\rangle\left(\frac{18\left(3 \cos ^{2} \beta-1\right)}{35}\right)}{1+\left\langle P_{2}\right\rangle\left(3 \cos ^{2} \beta-1\right)}-\alpha_{S S}\right] \exp \left(-t / \tau_{20}\right)+\alpha_{S S}
$$

and

$$
\alpha_{A S}=\frac{\frac{\sin ^{2} \beta}{5}\left(1-\frac{10}{7}\left\langle P_{2}\right\rangle+\frac{3}{7}\left\langle P_{4}\right\rangle\right)}{1+\left\langle P_{2}\right\rangle\left(3 \cos ^{2} \beta-1\right)} \exp \left(-t / \tau_{22}\right)
$$


Knowledge of the ground state order parameters and correction factors ${ }^{*}$ allows $\tau_{20}$ to be determined from the $\beta=0^{\circ}$ curve. Analysis of the $\beta=90^{\circ}$ curve a known value for $\tau_{20}$ is thus straightforward allowing $\tau_{22}$ to be determined. It is assumed in this step that $\theta$ diffusion (symmetric alignment relaxation) is independent of the initial molecular alignment. This is confirmed by the three beam excitation measurements where cylindrically symmetric alignment relaxation is seen to be independent of initial molecular orientation. From equations (3) and (4) the cylindrically symmetric and asymmetric alignment relaxation times can thus be used to determine the intrinsic $\theta$ and $\phi$ diffusion rates in the laboratory frame $\left(D_{\perp}^{L A B}\right.$ and $D_{\|}^{L A B}$ ), these are displayed in table 4 .

\begin{tabular}{|c|c|c|c|c|}
\hline$\tau_{20}(\mathrm{~ns})$ & $\tau_{22}(\mathrm{~ns})$ & $\gamma_{\theta} \equiv D_{\perp}^{L A B}\left(\times 10^{-7} \mathrm{~s}^{-1}\right)$ & $\gamma_{\phi} \equiv D_{\|}^{L A B}\left(\times 10^{-7} \mathrm{~s}^{-1}\right)$ & $\gamma_{\phi} / \gamma_{\theta}$ \\
\hline $12.06 \pm 1.15$ & $4.04 \pm 0.01$ & $1.3820 \pm 0.0007$ & $5.4971 \pm 0.0007$ & $3.98 \pm 0.02$ \\
\hline
\end{tabular}

Table 4. Values for the cylindrically symmetric and asymmetric relaxation times for oxazine 4 in $5 \mathrm{CB}$ together with the corresponding $\theta$ and $\phi$ diffusion rates relative to the nematic director (laboratory $\mathrm{Z}$ axis).

The anisotropy in the intrinsic $\theta$ and $\phi$ diffusion rates is marked with $\gamma_{\phi} \cong 4 \gamma_{\theta}$. The relative reduction in the $\theta$ diffusion rate is seen to go hand in hand with the restricted range of $\theta$ orientations the probe is allowed to adopt in the nematic host. Following the analysis ${ }^{15}$ of Durbin et al. the $\left\langle\mathrm{P}_{2}\right\rangle$ and $\left\langle\mathrm{P}_{4}\right\rangle$ order parameters can be used to determine the oxazine 4 ground state distribution. At $25^{\circ}$ this is seen to be sharply peaked at $\theta=40^{\circ}$ and $140^{\circ}$ to the nematic director with an approximate Gaussian width of $31^{\circ}$. Diffusion of the probe in the $\phi$ co-ordinate is unrestricted, the steady state value of all moments with $\mathrm{Q} \neq 0$ are necessarily zero in a cylindrically symmetric host.

A direct experimental verification of the difference in cylindrically symmetric and asymmetric relaxation is provided by a comparison between the evolution of the fluorescence anisotropy from cylindrically symmetric ( 3 beam excitation) and asymmetric molecular arrays (single beam excitation $\beta>0^{\circ}$ ) with the same initial anisotropy. Figure 5 shows that the range of initial anisotropy that can be created by single pulse photoselection covers the entire three beam tuning range. It is therefore possible to create an excited state via single photon photoselection with the same initial anisotropy as that created by three beam photoselection. The clearest case is provided by purely horizontal excitation $(\mathrm{B}(\mathrm{I})=-0.5)$ the corresponding single beam excited state will have the highest asymmetric alignment contribution. This offers a direct observation of the difference between cylindrically symmetric and asymmetric probe alignment relaxation, as shown in figure 7 .

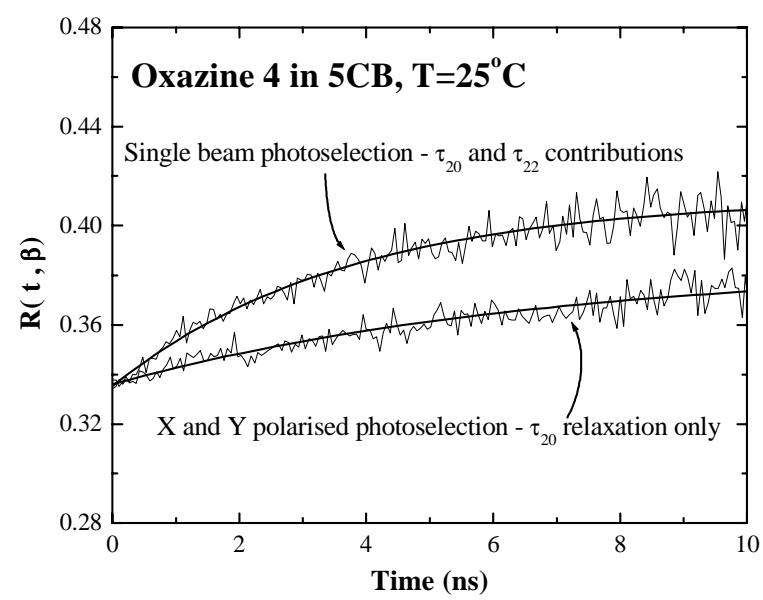

Figure 7. Fluorescence anisotropy evolution in oxazine 4 doped $5 \mathrm{CB}$, relaxation back to the steady state anisotropy $\left(\left\langle\mathrm{P}_{2}^{\mathrm{EX}}\right\rangle\right)$ of c.a. 0.37 is clearly more rapid in the case where the cylindrically asymmetric alignment is maximised.

${ }^{\ddagger}$ The inclusion of correction factors result in a correction to the lifetimes of c.a. $10 \%$ only. 


\section{CONCLUSIONS}

The combination of three and single pulse photoselection has proven to be a powerful tool in understanding the dynamics of a molecular probe in an ordered host. In the oxazine 4-5CB system in spite of the high degree of intrinsic probe order $\left(<\mathrm{P}_{2}{ }^{\mathrm{EX}}>\right.$ c.a. 0.37$)$ the alignment dynamics are seen to be wholly independent of the initial molecular orientation and conform well to first order relaxation processes as predicted by perturbation theory and simple symmetry considerations.

\section{Acknowledgements}

We would like to thank Adrian Bolan-Thomas (Essex University) for his generous help and assistance with aspects of the sample preparation. We would like to acknowledge ongoing support of this project by EPSRC.

\section{References}

[1] A J Bain, "Time Resolved Polarised Fluorescence Studies of Ordered Molecular Systems" in An Introduction to Laser Spectroscopy, Eds. D L Andrews and A Demidov (Kluwer 2002)

[2] A J Bain, P Chandna and G Butcher, Chem. Phys. Lett. 260, 441 (1996)

[3] A J Bain, P Chandna and J Bryant, J. Chem. Phys. 112, 10418 (2000)

[4] A J Bain, P Chandna, G Butcher and J Bryant, J. Chem. Phys. 112, 10435 (2000)

[5] A J Bain and J Bryant, Proc. of CLEO/QELS, paper QThC5 (1999)

[6] P S Pershan "Theory of Light Scattering", Proc. of the $1^{\text {st }}$ Joint USA-USSR Symposium, Plenum Press, Neyyor (1976)

[7] A J Bain and A J McCaffery, J. Chem. Phys. 80, 5893 (1984)

[8] A J Bain and A J McCaffery, J. Chem. Phys. 83, 2632 (1985)

[9] A J Bain and A J McCaffery, J. Chem. Phys. 83, 2641 (1985)

[10] Y Y Shen, "Principles of Nonlinear Optics", John Wiley and Sons (1984)

[11] N Chadborn, J Bryant, A J Bain and P O'Shea, Biophysics J. 76, 2198 (1999)

[12] A J Bain and J Bryant, Chem. Phys. Lett. 286, 121 (1998)

[13] J Shen, N A Clarke, P S Pershan and E B Priestly, J.Chem. Phys 66, 4635 (1977)

[14] J Bryant Ph.D. thesis, University of Essex (2000)

[15] S D Durbin, Y R Shen, Phys. Rev. A. 30, 1419 (1984)

[16] A J Bain, D A Armoogum and E M Monge, to be published

[17] Wu S-T, Wu C-S, Opt. Eng. 32, 1775 (1993)

[18] A J Bain, E M Monge, D A Armoogum, J Bryant and B Obradovic, Proc. of British Liquid Crystal Society Annual Meeting 2002 (unpublished)

[19] P R Hammond, J. Chem. Phys. 70, 3884 (1979)

[20] S Chandraesekhar, "Liquid Crystals", 2nd Ed. Cambridge University Press (1992) 